\title{
Efeito da Nifedipina Gel 0,2\% nas Pressões de Canal Anal e na Dor Pós-Operatória: Estudo após Hemorroidectomia pela Técnica Aberta
}

\author{
Effect of 0.2\% Nifedipine Gel on Anal Canal Pressures and Postoperative Pain: \\ Study Following Hemorrhoidectomy by Open Technique
}

\author{
MARIA AUXILIADORA PROLUNGATTICESAR ${ }^{1-2}$; WILMAR ARTUR KLUG ${ }^{1-3}$; DEOMIR GERMANO BASSI ${ }^{4-5}$; \\ PEDRO ROBERTO DE PAULA ${ }^{6-7}$; ROSANA PROLUNGATTICESAR ${ }^{8}$; JORGE ALBERTO ORTIZ ${ }^{9-10}$; \\ MANILIO BASILIO SPERANZINI ${ }^{11-12}$
}

\begin{abstract}
${ }^{1}$ Mestre e Doutor em Medicina pela FCMSCSP; ${ }^{2}$ Professor Assistente Doutor em Cirurgia da Universidade de Taubaté; ${ }^{3}$ Professor Titular em Cirurgia da FCMSCSP; ${ }^{4}$ Mestre e Doutor em Medicina pela Escola Paulista de Medicina; ${ }^{5}$

Professor Titular em Cirurgia da Universidade de Taubaté; ${ }^{6}$ Mestre e Doutor em Medicina pela Escola Paulista de Medicina $;{ }^{7}$ Professor Assistente e Doutor em Cirurgia da Universidade de Taubaté; ${ }^{8}$ Médico do Serviço de Endoscopia e Motilidade Digestiva do Hospital Universitário de Taubaté; ${ }^{9}$ Mestre em Medicina pela FCMSCSP; ${ }^{10}$ Responsavel pelo

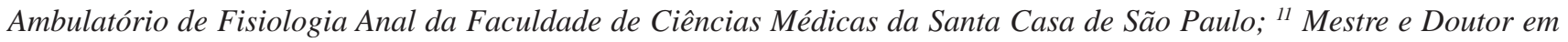
Medicina pela FMUSP; ${ }^{12}$ Professor Titular em Cirurgia da Faculdade de Medicina do ABC.
\end{abstract}

CESAR MAP; KLUG WA; BASSI DG; PAULA PR; CESAR RP; ORTIZ JA; SPERANZINI MB. Efeito da Nifedipina Gel 0,2\% nas Pressões de Canal Anal e na Dor Pós-Operatória: Estudo Após Hemorroidectomia pela Técnica Aberta. Rev bras Coloproct, 2007;27(4): 364-373.

RESUMO: Introdução: As hemorróidas são muito freqüentes e após o seu tratamento cirúrgico tem se observado que a dor causa muito sofrimento. Várias alternativas tem sido estudadas para melhorar a dor pós-operatória dentre elas a esfincterotomia cirúrgica que pode em alguns casos causar algum grau de incontinência fecal. Por esse motivo vários estudos tem utilizado a esfincterotomia química com nifedipina, diltiazen, trinitrato de glicerina e toxina botulínica. O objetivo dessa pesquisa foi avaliar o efeito da nifedipina tópica nas diminuições das pressões do canal anal e consequente influência na melhora da dor pósoperatória. Material e Método: Utilização da nifedipina tópica gel 0,2\% (Grupo 1) e lidocaina $2 \%$ (Grupo 2) no pós operatório de hemorroidectomia aferindo as pressões no pré, primeiro, quarto e sétimo dias de pós operatório, associado de medida de dor todos os dias do pós-operatório através de tabela analógica. Resultados: Os autores não encontraram diferenças em relação às pressões de canal anal mas em relação à dor referida estas foram em menor intensidade no grupo que recebeu a nifedipina. Conclusões - a nifedipina gel foi eficiente na analgesia pós-operatória, no entanto não alterou as pressões do canal anal.

Descritores: Nifedipino, Manometria, Canal anal, Hemorróidas/cirurgia, Dor pós-operatória, procedimentos cirúrgicos operatórios.

\section{INTRODUÇÃO}

A dor representa o maior inconveniente no pósoperatório das hemorroidectomias, sendo proporcional aos cuidados técnicos e ao preparo psicológico préoperatório do paciente $(1,2,3)$.
$\mathrm{Na}$ gênese da dor após hemorroidectomia a causa não é bem definida, podendo ser relacionada ao espasmo do esfincter interno do ânus e talvez também do esfincter externo, sendo que a dor intensa no momento da evacuação poderia manter o espasmo $(2,3,4)$. Outros autores opinam pelo contrário e acham que o 
espasmo esfincteriano não seria relevante na gênese da dor (1). O objetivo deste trabalho foi a avaliação das pressões anais e dor pós-operatória de pacientes submetidos à hemorroidectomia pela técnica aberta $\mathrm{e}$ uso da nifedipina gel $0,2 \%$, comparados com grupo controle para observação do efeito da nifedipina gel na melhora da dor pós-operatória sob forma de esfincterotomia química .

\section{CASUÍSTICA E MÉTODO}

Realizou-se estudo prospectivo, onde foram avaliadas as pressões do canal anal (repouso, contração e esforço evacuatório) e índices de dor no pré e pós-operatório de hemorroidectomia com o uso tópico de nifedipina gel $0,2 \%$ + lidocaina gel $2 \%$ comparados com o grupo controle com o uso tópico de lidocaina gel $2 \%$. Todos os pacientes que participaram da pesquisa se submeteram à hemorroidectomia e eram pacientes do Hospital Universitário de Taubaté - Serviço de Clínica Cirúrgica,no período de abril de 2005 a janeiro de 2006.

Todos os pacientes analisados no presente estudo eram, antes da execução do exame, orientados a assinarem um Termo de Consentimento Livre e Esclarecido, concordando com a participação. Tal instrumento de pesquisa teve sua apreciação e aprovação na Comissão de Ética Médica (CEM) do Hospital Universitário de Taubaté e pela Comissão de Ética em Pesquisa da Universidade de Taubaté (CEP-UNITAU) (em anexo).

Para o estudo os pacientes foram divididos em dois grupos aleatórios não identificados previamente :

1 - grupo 1 - utilização da nifedipina $0,2 \%$ gel associada com lidocaina $2 \%$ gel quatro vezes ao dia na região perianal por 30 dias

2 - grupo 2 - utilização da lidocaina $2 \%$ gel quatro vezes ao dia na região perianal por 30 dias

Foram excluídos da amostra pacientes que se submeteram as cirurgias prévias anos-retais ; presença de doença ano-retal concomitante e uso de antihipertensivo via oral. Para o procedimento cirúrgico os pacientes receberam bloqueio raquidiano, sendo realizada a hemorroidectomia aberta pela técnica de Milligan-Morgan. No pós-operatório orientação higienodietética, diclofenaco de sódio $50 \mathrm{mg}$ um comprimido via oral a cada oito horas por cinco dias, dipirona 30 gotas via oral a cada oito horas por sete dias e ingestão de 200 g de fibra dietética uma vez ao dia e realização de banhos de assento por 20 minutos quatro vezes ao dia seguido da aplicação do gel nifedipina + lidocaina (Grupo 1) ou lidocaina (Grupo 2) a partir do primeiro pós-operatório .

O estudo manométrico foi realizado com o aparelho portátil Proctosystem PL-3000 e era realizado sem preparo intestinal prévio nos momentos pré-operatório , primeiro ,quarto e sétimo dias de pós-operatório. O paciente permanecia em decúbito lateral esquerdo e o cateter era introduzido até 5 centímetros e então retirado a cada centímetro registrando pressão máxima de repouso (PMR); pressão máxima de contração (PMC) e pressão mínima de evacuação (PME).

A dor anal era avaliada com uma escala analógica de 0-10 (nenhuma dor, nota zero; máximo de dor, nota 10 - a maior dor que possa ter experimentado), que era anotada pelo paciente às $8 \mathrm{~h}, 20 \mathrm{~h}$ e após as evacuações do primeiro ao sétimo dia de pós-operatório.

Para a análise estatística usamos o programa EPI INFO, para a obtenção dos resultados e adotamos o nível de significância de $5 \%$.

Foram aplicados os Testes de Mann-Whitney e ANOVA, com o intuito de verificar-se possíveis diferenças dos valores das demais variáveis entre os Grupos 1 e 2 .

\section{RESULTADOS}

Foram estudados 16 pacientes, cuja idade variou de 25 a 50 anos no grupo 1, média de 41,25 $\pm 10,28$ anos em relação à média de 46,25 \pm 6,67 no grupo 2 , não existindo diferença estatística

Não encontramos diferenças entre os valores de manometria anal no pré-operatório e no primeiro pós-operatório entre os dois grupos, momento em que os pacientes tinham se submetido a um mesmo evento.

As médias, mediana, valor mínimo, valor máximo, valores estatísticos pelos métodos Anova e Man Witney das pressões de repouso, contração e evacuação aferidas no quarto pós-operatório encontram-se dispostas nas Tabelas de 1-3, e não apresentaram diferenças estatisticamente significantes mesmo no momento em que o grupo 1 já esta recebendo a nifedipina tópica há 3 dias.

As médias, mediana, valor mínimo, valor máximo, valores estatísticos pelos métodos Anova e Man Witney das pressões de repouso, contração e evacuação aferidas no pós-operatório sete dias encontramse dispostas nas Tabelas de 4-6. 
Tabela 1 - Medidas das pressões de repouso nos Grupos 1 e 2 segundo a altura do canal anal no quarto dia de pós-operatório.

\begin{tabular}{lccccrrrrrr}
\hline Variável & Dist. Grupo & N & Média & DP & Mínima & Mediana & Máxima & \multicolumn{2}{c}{ N. Descritivo (P) } \\
& & & & & & & & & Anova & M-W \\
\hline Pressão & 5 & 1 & 8 & 30,1250 & 21,0132 & 8,0000 & 24,0000 & 70,0000 & 0,7644 & 0,9164 \\
& & 2 & 8 & 27,6250 & 9,6797 & 15,0000 & 26,0000 & 43,0000 & & \\
de & 4 & 1 & 8 & 25,3750 & 19,0483 & 6,0000 & 19,0000 & 66,0000 & 0,4139 & 0,3439 \\
& & 2 & 8 & 32,6250 & 15,1746 & 12,0000 & 31,5000 & 54,0000 & & \\
Repouso & \multirow{2}{*}{3} & 1 & 8 & 39,5000 & 26,4683 & 10,0000 & 30,5000 & 94,0000 & 0,1682 & 0,1033 \\
& & 2 & 8 & 58,8750 & 26,8617 & 13,0000 & 63,0000 & 100,0000 & & \\
& 2 & 1 & 8 & 57,6250 & 23,3601 & 20,0000 & 62,5000 & 98,0000 & 0,7743 & 0,3442 \\
& & 2 & 8 & 60,8750 & 21,0471 & 24,0000 & 69,0000 & 85,0000 & & \\
& 1 & 1 & 8 & 55,7500 & 14,7430 & 35,0000 & 54,5000 & 85,0000 & 0,5260 & 0,3431 \\
& & 2 & 8 & 62,5000 & 25,3828 & 28,0000 & 64,5000 & 112,0000 & & \\
\hline
\end{tabular}

Tabela 2 - Medidas das pressões de contração nos Grupos 1 e 2 segundo a altura do canal anal no quarto dia de pós-operatório.

\begin{tabular}{lcccccccccc}
\hline Variável & Dist. Grupo & N & Média & DP & Mínima & Mediana & Máxima & \multicolumn{2}{c}{$\begin{array}{c}\text { N. Descritivo (P) } \\
\text { Anova }\end{array}$} & M-W \\
\hline Pressão & 5 & 1 & 8 & 40,5000 & 26,1370 & 7,0000 & 36,5000 & 90,0000 & 0,4127 & 0,3717 \\
& & 2 & 8 & 50,2500 & 19,5941 & 24,0000 & 50,0000 & 85,0000 & & \\
de & 4 & 1 & 8 & 51,8750 & 36,3925 & 11,0000 & 43,5000 & 122,0000 & 0,3643 & 0,2698 \\
& & 2 & 8 & 66,7500 & 26,2393 & 23,0000 & 71,5000 & 103,0000 & & \\
Contração & \multirow{2}{*}{3} & 1 & 8 & 75,8750 & 47,8523 & 17,0000 & 62,0000 & 155,0000 & 0,2905 & 0,3717 \\
& & 2 & 8 & 103,3750 & 52,1836 & 47,0000 & 102,5000 & 198,0000 & & \\
& 2 & 1 & 8 & 124,2500 & 67,9848 & 31,0000 & 104,0000 & 230,0000 & 0,9970 & 0,7527 \\
& & 2 & 8 & 124,3750 & 64,2071 & 37,0000 & 120,5000 & 220,0000 & & \\
& \multirow{2}{*}{1} & 1 & 8 & 131,1250 & 48,6311 & 69,0000 & 112,0000 & 213,0000 & 0,8432 & 1,0000 \\
& & 2 & 8 & 125,3750 & 64,3915 & 40,0000 & 130,5000 & 229,0000 & & \\
\hline
\end{tabular}

Tabela 3 - Medidas das pressões de evacuação nos Grupos 1 e 2 segundo a altura do canal anal no quarto dia de pós-operatório.

\begin{tabular}{|c|c|c|c|c|c|c|c|c|c|c|}
\hline \multirow[t]{2}{*}{ Variável } & \multirow{2}{*}{\multicolumn{2}{|c|}{ Dist. Grupo }} & \multirow[t]{2}{*}{$\mathbf{N}$} & \multirow[t]{2}{*}{ Média } & \multirow[t]{2}{*}{ DP } & \multirow[t]{2}{*}{ Mínima } & \multirow[t]{2}{*}{ Mediana } & \multirow[t]{2}{*}{ Máxima } & \multicolumn{2}{|c|}{ N. Descritivo (P) } \\
\hline & & & & & & & & & Anova & $\mathbf{M}-\mathbf{W}$ \\
\hline \multirow[t]{2}{*}{ Pressão } & 5 & 1 & 8 & 43,5000 & 27,3130 & 15,0000 & 43,0000 & 98,0000 & 0,3822 & 0,2694 \\
\hline & & 2 & 8 & 54,5000 & 21,0510 & 29,0000 & 52,0000 & 95,0000 & & \\
\hline \multirow[t]{2}{*}{ de } & 4 & 1 & 8 & 50,3750 & 35,5244 & 14,0000 & 41,5000 & 120,0000 & 0,9730 & 0,4945 \\
\hline & & 2 & 8 & 50,8750 & 20,6635 & 33,0000 & 41,5000 & 90,0000 & & \\
\hline \multirow[t]{6}{*}{ Evacuação } & 3 & 1 & 8 & 48,8750 & 21,3972 & 31,0000 & 40,0000 & 99,0000 & 0,8216 & 0,5984 \\
\hline & & 2 & 8 & 51,1250 & 17,5779 & 33,0000 & 47,5000 & 81,0000 & & \\
\hline & 2 & 1 & 8 & 58,2500 & 27,3744 & 34,0000 & 52,5000 & 120,000 & 0,5820 & 0,9580 \\
\hline & & 2 & 8 & 52,1250 & 13,9943 & 30,0000 & 52,5000 & 74,0000 & & \\
\hline & 1 & 1 & 8 & 42,1250 & 12,4147 & 28,0000 & 41,5000 & 69,0000 & 0,8929 & 1,000 \\
\hline & & 2 & 8 & 43,0000 & 13,0931 & 28,0000 & 39,5000 & 64,0000 & & \\
\hline
\end{tabular}



Maria Auxiliadora Prolungatti Cesar e Cols.

Tabela 4 - Medidas das pressões de repouso nos Grupos 1 e 2 segundo a altura do canal anal no sétimo dia de pós-operatório.

\begin{tabular}{lcccccrrrrr}
\hline Variável & Dist. Grupo & N & Média & DP & Mínima & Mediana & Máxima & \multicolumn{2}{c}{ N. Descritivo (P) } \\
& & & & & & & & & Anova & M-W \\
\hline Pressão & 5 & 1 & 8 & 28,5000 & 26,7368 & 6,0000 & 19,0000 & 90,0000 & 0,5873 & 0,5280 \\
& & 2 & 8 & 23,0000 & 8,3324 & 10,0000 & 24,0000 & 33,0000 & & \\
de & 4 & 1 & 8 & 25,1250 & 20,6566 & 6,0000 & 15,0000 & 68,0000 & 0,2090 & 0,1250 \\
& & 2 & 8 & 37,5000 & 16,7247 & 13,0000 & 39,5000 & 66,0000 & & \\
Repouso & \multirow{2}{*}{3} & 1 & 8 & 30,3750 & 21,9150 & 8,0000 & 23,5000 & 71,0000 & 0,0295 & 0,0587 \\
& & 2 & 8 & 60,8750 & 28,0583 & 2,0000 & 65,0000 & 91,0000 & & \\
& 2 & 1 & 8 & 61,5000 & 28,3246 & 30,0000 & 57,0000 & 124,0000 & 0,8701 & 0,3710 \\
& & 2 & 8 & 59,5000 & 18,7236 & 17,0000 & 64,0000 & 77,0000 & & \\
& \multirow{2}{*}{1} & 1 & 8 & 51,8750 & 10,1621 & 30,0000 & 54,0000 & 62,0000 & 0,2292 & 0,1136 \\
& & 2 & 8 & 59,1250 & 12,7553 & 34,0000 & 62,0000 & 71,0000 & & \\
\hline
\end{tabular}

Tabela 5 - Medidas das pressões de contração nos Grupos 1 e 2 segundo a altura do canal anal no sétimo dia de pós-operatório.

\begin{tabular}{lcccccccccc}
\hline Variável & Dist. Grupo & N & Média & DP & Mínima & Mediana & Máxima & \multicolumn{2}{c}{ N. Descritivo (P) } \\
& & & & & & & & & Anova & M-W \\
\hline Pressão & 5 & 1 & 8 & 34,6250 & 33,5045 & 5,0000 & 21,0000 & 104,0000 & 0,2165 & 0,1149 \\
& & 2 & 8 & 52,5000 & 20,0855 & 21,0000 & 56,0000 & 87,0000 & & \\
de & 4 & 1 & 8 & 47,1250 & 43,4263 & 5,0000 & 24,5000 & 118,0000 & 0,1859 & 0,1722 \\
& & 2 & 8 & 75,8750 & 39,1351 & 37,0000 & 74,0000 & 146,0000 & & \\
Contração & \multirow{2}{*}{3} & 1 & 8 & 95,1250 & 52,9702 & 19,0000 & 119,0000 & 164,0000 & 0,4323 & 0,3181 \\
& & 2 & 8 & 116,0000 & 50,2679 & 48,0000 & 124,5000 & 187,0000 & & \\
& 2 & 1 & 8 & 151,3750 & 42,2609 & 90,0000 & 142,0000 & 236,0000 & 0,6361 & 0,6744 \\
& & 2 & 8 & 137,8750 & 66,6814 & 31,0000 & 130,0000 & 213,0000 & & \\
& \multirow{2}{*}{1} & 1 & 8 & 138,8750 & 37,9791 & 85,0000 & 123,0000 & 189,0000 & 0,7609 & 0,7527 \\
& & 2 & 8 & 131,2500 & 58,2108 & 34,0000 & 133,5000 & 231,0000 & & \\
\hline
\end{tabular}

Tabela 6 - Medidas das pressões de evacuação nos Grupos 1 e 2 segundo a altura do canal anal no sétimo dia de pós-operatório.

\begin{tabular}{lcccccrrrrr}
\hline Variável & Dist. Grupo & N & Média & DP & Mínima & Mediana & Máxima & \multicolumn{2}{c}{ N. Descritivo (P) } \\
& & & & & & & & & Anova & M-W \\
\hline Pressão & 5 & 1 & 8 & 41,3750 & 27,5988 & 8,0000 & 33,0000 & 99,0000 & 0,8144 & 0,5982 \\
& & 2 & 8 & 44,0000 & 14,1825 & 20,0000 & 46,0000 & 64,0000 & & \\
& 4 & 1 & 8 & 41,5000 & 22,7031 & 6,0000 & 39,0000 & 85,0000 & 0,7994 & 0,4606 \\
& & 2 & 8 & 43,7500 & 9,4074 & 28,0000 & 43,5000 & 59,0000 & & \\
& 3 & 1 & 8 & 45,7500 & 20,7898 & 13,0000 & 45,0000 & 72,0000 & 0,8766 & 0,9162 \\
& 2 & 2 & 8 & 47,1250 & 13,1523 & 33,0000 & 45,5000 & 70,0000 & & \\
& 2 & 1 & 8 & 56,5000 & 24,4949 & 40,0000 & 48,5000 & 116,0000 & 0,3072 & 1,0000 \\
& & 2 & 8 & 44,3750 & 21,1453 & 8,0000 & 52,5000 & 70,0000 & & \\
& 1 & 1 & 8 & 46,1250 & 12,0882 & 31,0000 & 46,0000 & 69,0000 & 0,1067 & 0,1028 \\
& & 2 & 8 & 35,5000 & 12,5584 & 20,0000 & 35,0000 & 58,0000 & & \\
\hline
\end{tabular}


Observamos que as pressões de repouso também não apresentaram diferenças estatisticamente significantes, apesar de que a pressão de repouso a 3 $\mathrm{cm}$ foi muito próxima da diferença estatística $(\mathrm{p}=0,059)$ pelo teste de mann-witney e diferente pelo teste de anova, sendo a média do grupo 2 maior que o dobro da média do grupo 1 .

Não houve diferença entre os grupos no pré e pós-operatório no período da manhã quando comparados em relação à dor .

A dor referida no primeiro dia de pós-operatório às $8 \mathrm{~h}, 20 \mathrm{~h}$ e após as evacuações nos Grupos 1 e 2 encontram-se dispostas na Tabela 7 e observamos diferenças significantes entre os 2 grupos sendo a dor no primeiro pós-operatório no período noturno menor no grupo que usou a nifedipina . Já no segundo pós-operatório podemos observar que a dor referida tanto no período da manhã como no período da noite foi estatisticamente menor no grupo 1. A dor após a evacuação não apresentou diferença entre os 2 grupos (Tabela 8).

No segundo pós-operatório podemos observar que a dor referida tanto no período da noite como no período da manhã foi menor no Grupo 1. A dor após evacuação embora menor no grupo 1 não apresentou diferença significativa entre os dois grupos (Tabela 9).

Os valores de dor no terceiro pós-operatório estão expostos na Tabela 9 e mostram que os valores de dor no período da manhã foram menores no grupo 1 e ocorreu de maneira significante $(\mathrm{p}=0,001)$, sendo que o grupo que estava usando a nifedipina gel $0,2 \%$ referiu menor intensidade de dor.

No quarto dia de pós-operatório observamos dor de menor intensidade no período da noite no grupo que recebeu a nifedipina gel com lidocaina (Tabela 10).

No quinto dia de pós-operatório observamos que a os pacientes do grupo que estava recebendo a nifedipina gel apresentaram menores índices de dor de manhã, à noite e após as evacuações (Tabela 11).

No sexto dia de pós-operatório observamos que a dor pós-operatória no período da noite foi significantemente menor no grupo que usou a nifedipina gel , já a dor no período da manhã e após as evacuações apresentaram valores diferentes, porém sem significância estatística (Tabela 12).

Tabela 7 - Valores de dor atribuídos pelos pacientes na tabela analógica nos períodos da manhã (8h), noite $(20$ h) e após a evacuação no primeiro dia de pós-operatório.

\begin{tabular}{lcccccccccc}
\hline Variável & Tempo Grupo & N & Média & DP & Mínima & Mediana & Máxima & \multicolumn{2}{c}{ N. Descritivo (P) } \\
& & & & & & & & & Anova & M-W \\
\hline Momento & \multirow{2}{*}{$\mathrm{h}$} & 1 & 8 & 3,3750 & 1,5059 & 1,0000 & 3,0000 & 5,0000 & 0,3702 & 0,3215 \\
da dor & & 2 & 8 & 4,1250 & 1,7269 & 1,0000 & 5,0000 & 6,0000 & & \\
& \multirow{2}{*}{$20 \mathrm{~h}$} & 1 & 8 & 3,7500 & 1,3887 & 1,0000 & 4,0000 & 5,0000 & 0,0281 & 0,0338 \\
& & 2 & 8 & 5,2500 & 1,0351 & 4,0000 & 5,0000 & 7,0000 & & \\
& \multirow{2}{*}{ Pós-ev. } & 1 & 8 & 8,4000 & 1,5166 & 7,0000 & 8,0000 & 10,0000 & 0,4653 & 0,5676 \\
& & 2 & 8 & 9,0000 & 1,0954 & 7,0000 & 9,0000 & 10,0000 & & \\
\hline
\end{tabular}

Tabela 8 - Valores de dor atribuídos pelos pacientes na tabela analógica nos períodos da manhã (8h), noite $(20$ h) e após a evacuação no segundo dia de pós-operatório.

\begin{tabular}{|c|c|c|c|c|c|c|c|c|c|c|}
\hline \multirow[t]{2}{*}{ Variável } & \multirow[t]{2}{*}{ Tempo } & \multirow[t]{2}{*}{ Grupo } & \multirow[t]{2}{*}{$\mathbf{N}$} & \multirow[t]{2}{*}{ Média } & \multirow[t]{2}{*}{ DP } & \multirow[t]{2}{*}{ Mínima } & \multirow[t]{2}{*}{ Mediana } & \multirow[t]{2}{*}{ Máxima } & \multicolumn{2}{|c|}{ N. Descritivo $(\mathbf{P})$} \\
\hline & & & & & & & & & Anova & M-W \\
\hline \multirow{6}{*}{$\begin{array}{l}\text { Momento } \\
\text { da dor }\end{array}$} & \multirow[t]{2}{*}{$8 \mathrm{~h}$} & 1 & 8 & 2,7500 & 2,1213 & 1,0000 & 2,0000 & 7,0000 & \multirow[t]{2}{*}{0,0386} & \multirow[t]{2}{*}{0,0436} \\
\hline & & 2 & 8 & 4,7500 & 1,2817 & 3,0000 & 5,0000 & 6,0000 & & \\
\hline & \multirow[t]{2}{*}{$20 \mathrm{~h}$} & 1 & 8 & 3,8750 & 0,9910 & 2,0000 & 4,0000 & 5,0000 & \multirow[t]{2}{*}{0,0083} & \multirow[t]{2}{*}{0,0120} \\
\hline & & 2 & 8 & 5,8750 & 1,5526 & 3,0000 & 6,0000 & 8,0000 & & \\
\hline & \multirow[t]{2}{*}{ Pós-ev. } & 1 & 8 & 6,2000 & 2,3875 & 3,0000 & 6,0000 & 9,0000 & \multirow[t]{2}{*}{0,0775} & \multirow[t]{2}{*}{0,1340} \\
\hline & & 2 & 8 & 8,3333 & 1,0328 & 7,0000 & 8,0000 & 10,0000 & & \\
\hline
\end{tabular}


Tabela 9 - Valores de dor atribuídos pelos pacientes na tabela analógica nos períodos da manhã (8h), noite $(20$ h) e após a evacuação no terceiro dia de pós-operatório.

\begin{tabular}{lcccccccrcc}
\hline Variável & Tempo Grupo & N & Média & DP & Mínima & Mediana & Máxima & \multicolumn{2}{c}{ N. Descritivo (P) } \\
& & & & & & & & & Anova & M-W \\
\hline Momento & \multirow{2}{*}{$\mathrm{h}$} & 1 & 8 & 2,3750 & 1,4079 & 1,0000 & 2,5000 & 5,0000 & 0,0002 & 0,0012 \\
da dor & & 2 & 8 & 6,3750 & 1,7678 & 4,0000 & 6,0000 & 10,0000 & & \\
& \multirow{2}{*}{ 20h } & 1 & 8 & 3,5000 & 2,0702 & 1,0000 & 3,0000 & 8,0000 & 0,0445 & 0,0323 \\
& & 2 & 8 & 5,5000 & 1,5119 & 3,0000 & 6,0000 & 8,0000 & & \\
& \multirow{2}{*}{ Pós-ev. } & 1 & 8 & 6,1667 & 1,8348 & 5,0000 & 5,0000 & 9,0000 & 0,3018 & 0,3478 \\
& & 2 & 8 & 7,6000 & 2,5100 & 4,0000 & 7,0000 & 10,0000 & & \\
\hline
\end{tabular}

Tabela 10 - Valores de dor atribuídos pelos pacientes na tabela analógica nos períodos da manhã (8h), noite $(20$ h) e após a evacuação no quarto dia de pós-operatório.

\begin{tabular}{lcccccccccc}
\hline Variável & Tempo Grupo & N & Média & DP & Mínima & Mediana & Máxima & \multicolumn{2}{c}{ N. Descritivo (P) } \\
& & & & & & & & & Anova & M-W \\
\hline Momento & \multirow{2}{*}{ h h } & 1 & 8 & 3,1250 & 1,5526 & 1,0000 & 4,0000 & 5,0000 & 0,2991 & 0,3567 \\
da dor & & 2 & 8 & 4,0000 & 1,6903 & 1,0000 & 4,0000 & 6,0000 & & \\
& \multirow{2}{*}{ 20h } & 1 & 8 & 3,1250 & 1,2464 & 1,0000 & 3,0000 & 5,0000 & 0,0047 & 0,0051 \\
& & 2 & 8 & 5,2500 & 1,2817 & 4,0000 & 5,0000 & 8,0000 & & \\
& \multirow{2}{*}{ Pós-ev } & 1 & 8 & 4,7143 & 2,5635 & 2,0000 & 4,0000 & 10,0000 & 0,2271 & 0,1375 \\
& & 2 & 8 & 6,6000 & 2,4083 & 4,0000 & 6,0000 & 10,0000 & & \\
\hline
\end{tabular}

Os valores no sétimo pós-operatório encontram-se na Tabela 13 , encontramos diferença estatística na dor referida pelos pacientes nos dois a dor após a evacuação foi menor, mas a diferença não foi significante.

\section{DISCUSSÃO}

A dor é fator presente no pós-operatório de hemorroidectomia, implicando em sofrimento, princi- palmente nos momentos que se sucedem ao movimento evacuatório devido à sua intensidade e provável espasmo esfincteriano.

Alguns estudos demonstram que a hemorroidectomia isoladamente não altera as pressões de canal anal e que não ocorrem repercussões funcionais ou morfológicas no esfíncter interno após o procedimento $(5,6,7)$. Em estudos manométricos realizados no Hospital Universitário de Taubaté em pacientes portadores de hemorróidas no pré e pós-operatório 1 mês

Tabela 11 - Valores de dor atribuídos pelos pacientes na tabela analógica nos períodos da manhã (8h), noite $(20$ h) e após a evacuação no quinto dia de pós-operatório.

\begin{tabular}{lcccccccrcc}
\hline Variável & Tempo Grupo & N & Média & DP & Mínima & Mediana & Máxima & \multicolumn{2}{c}{ N. Descritivo (P) } \\
& & & & & & & & & & \\
Anova & M-W \\
\hline Momento & \multirow{2}{*}{$\mathrm{h}$} & 1 & 8 & 2,3750 & 1,0607 & 1,0000 & 2,5000 & 4,0000 & 0,0014 & 0,0012 \\
da dor & & 2 & 8 & 5,6250 & 2,0659 & 4,0000 & 5,0000 & 10,0000 & & \\
& \multirow{2}{*}{ 20h } & 1 & 8 & 2,7500 & 1,2817 & 1,0000 & 3,0000 & 4,0000 & 0,0006 & 0,0019 \\
& & 2 & 8 & 6,3750 & 1,9226 & 4,0000 & 6,5000 & 10,0000 & & \\
& \multirow{2}{*}{ Pós-ev } & 1 & 8 & 5,2500 & 0,5000 & 5,0000 & 5,0000 & 6,0000 & 0,0045 & 0,0090 \\
& & 2 & 8 & 8,1429 & 1,4639 & 6,0000 & 8,0000 & 10,0000 & & \\
\hline
\end{tabular}


Tabela 12 - Valores de dor atribuídos pelos pacientes na tabela analógica nos períodos da manhã (8h), noite $(20$ h) e após a evacuação no sexto dia de pós-operatório.

\begin{tabular}{lcccccccccc}
\hline Variável & Tempo Grupo & N & Média & DP & Mínima & Mediana & Máxima & \multicolumn{2}{c}{ N. Descritivo (P) } \\
& & & & & & & & & Anova & M-W \\
\hline Momento & \multirow{2}{*}{$\mathrm{h}$} & 1 & 8 & 2,2500 & 1,0351 & 1,0000 & 2,0000 & 4,0000 & 0,0288 & 0,0550 \\
da dor & & 2 & 8 & 4,7500 & 2,7124 & 1,0000 & 5,0000 & 9,0000 & & \\
& \multirow{2}{*}{ 20h } & 1 & 8 & 2,2500 & 1,2817 & 1,0000 & 2,0000 & 4,0000 & 0,0072 & 0,0093 \\
& & 2 & 8 & 5,3750 & 2,5036 & 2,0000 & 5,5000 & 10,0000 & & \\
& \multirow{2}{*}{ Pós-ev } & 1 & 8 & 5,2500 & 2,4928 & 3,0000 & 4,5000 & 10,0000 & 0,0596 & 0,0557 \\
& & 2 & 8 & 7,5000 & 1,8516 & 5,0000 & 7,5000 & 10,0000 & & \\
\hline
\end{tabular}

Tabela 13 - Valores de dor atribuídos pelos pacientes na tabela analógica nos períodos da manhã (8h), noite $(20$ h) e após a evacuação no sétimo dia de pós-operatório.

\begin{tabular}{|c|c|c|c|c|c|c|c|c|c|c|}
\hline \multirow[t]{2}{*}{ Variável } & \multirow[t]{2}{*}{ Tempo } & \multirow[t]{2}{*}{ Grupo } & \multirow[t]{2}{*}{$\mathbf{N}$} & \multirow[t]{2}{*}{ Média } & \multirow[t]{2}{*}{ DP } & \multirow[t]{2}{*}{ Mínima } & \multirow[t]{2}{*}{ Mediana } & \multirow[t]{2}{*}{ Máxima } & \multicolumn{2}{|c|}{ N. Descritivo (P) } \\
\hline & & & & & & & & & Anova & M-W \\
\hline \multirow{6}{*}{$\begin{array}{l}\text { Momento } \\
\text { da dor }\end{array}$} & $8 \mathrm{~h}$ & 1 & 8 & 2,2500 & 1,4880 & 1,0000 & 2,0000 & 5,0000 & 0,0125 & 0,0210 \\
\hline & & 2 & 8 & 5,1250 & 2,4165 & 1,0000 & 6,0000 & 8,0000 & & \\
\hline & $20 \mathrm{~h}$ & 1 & 8 & 2,3750 & 1,4079 & 1,0000 & 2,5000 & 5,0000 & 0,0082 & 0,0045 \\
\hline & & 2 & 8 & 5,5000 & 2,5071 & 4,0000 & 4,0000 & 10,0000 & & \\
\hline & Pós-ev & 1 & 8 & 4,0000 & 1,0000 & 3,0000 & 4,0000 & 5,0000 & 0,1191 & 0,0957 \\
\hline & & 2 & 8 & 6,1667 & 2,6394 & 2,0000 & 6,5000 & 10,0000 & & \\
\hline
\end{tabular}

os autores observaram não haver alteração nas pressões e no comprimento do canal anal em pacientes submetidos à hemorroidectomia pela técnica aberta (7). Em outro estudo se observou que a pressão de repouso nos pacientes com doença hemorroidária é maior que no grupo controle, mas que, após a cirurgia, existe um decréscimo da pressão de repouso (8).

Vários procedimentos tem sido utilizados na tentativa de melhora da dor pós-operatória , seja mudando a técnica cirúrgica (hemorroidectomia fechada ou grampeamento), seja associando a esfincterotomia , química ou cirúrgica. A esfincterotomia cirúrgica é defendida por alguns como alternativa para a melhora da dor após hemorroidectomia , podendo ser realizada em pacientes que apresentam pressão máxima de repouso aumentada no pré-operatório $(5,9,10)$.

O mecanismo em que a esfincterotomia cirúrgica ou química aliviam a dor e o desconforto pós-operatório, porém, permanecem sem resposta (2) e se sabe que algum grau de alteração funcional pode ocorrer, principalmente relacionadas à continência fecal (11; $2 ; 12 ; 13 ; 14)$.
Em outros estudos manométricos no pré e pósoperatório em que alguns pacientes apresentavam hipertonia esfincteriana no pré-operatório e queda das pressões no pós-operatório os autores concluem que a esfincterotomia cirúrgica deveria ser utilizada associada ao tratamento cirúrgico das hemorróidas $(9,10)$.

Com a mesma opinião outros autores investigaram os achados manométricos após o tratamento cirúrgico das hemorróidas combinado com a esfincterotomia em estudo prospectivo com 48 pacientes, tendo dez voluntários como controle. A manometria foi realizada duas vezes, e foram encontradas alterações esfincterianas em $87,5 \%$ dos pacientes. Observaram que a hemorroidectomia, isoladamente, não alterou as pressões anais, e no pós-operatório foi com menor dor no grupo com esfincterotomia e não ocorreu incontinência. Este estudo demonstrou que pressões altas são muito comuns em pacientes com hemorróidas e estes pacientes deveriam submeter-se à hemorroidectomia com esfincterotomia desde que confirmada essa hipertonia, pois melhora o curso pósoperatório relacionado com a dor (5). 
A realização da esfincterotomia cirúrgica não é consenso na literatura. Em estudo manométrico de pacientes submetidos à hemorroidectomia com ou sem esfincterotomia, não foram encontradas diferenças nas pressões de repouso e contração no pós-operatório de seis semanas e de três meses e afirmaram que no grupo da esfincterotomia as pressões foram mais baixas, mas não houve diferença significante. Foi observado nessa pesquisa que dois pacientes, no grupo da esfincterotomia, apresentaram incontinência para fezes líquidas no pós-operatório, e em um deles essa incontinência persistiu depois de um ano. Concluíram que a esfincterotomia foi desnecessária e que ela implicaria no risco adicional de incontinência fecal.15

Devido à possibilidade de lesão esfincteriana após a esfincterotomia cirúrgica passou-se a utilizar os nitratos orgânicos e bloqueadores de canal de cálcio com a finalidade de reduzir a hipertonia esfincteriana, melhorar o fluxo sanguíneo da mucosa e promover cicatrização no tratamento da fissura anal crônica e atualmente no pós-operatório de hemorroidectomia (16; $17 ; 18 ; 19)$.

Comparando a efetividade da esfincterotomia química e cirúrgica para reduzir a dor póshemorroidectomia se observou que o grupo submetido à esfincterotomia cirúrgica apresentou menor dor pósoperatória e menor grau de estenose, não tendo havido, nessa casuística, nenhum caso de incontinência, mesmo leve.(3)

$\mathrm{Na}$ tentativa de buscar alternativas para alívio da dor após hemorroidectomia foi realizada análise quatro horas após a cirurgia, após o primeiro movimento evacuatório, e quatro dias depois. Não foram encontradas diferenças estatisticamente significantes, nos grupos que se submeteram à hemorroidectomia, com ou sem esfincterotomia, definindo esse procedimento como desnecessário se o objetivo é dor. Também, não encontrou alterações quando estudou o controle de gás ou soiling .

Utilizando a esfincterotomia química após hemorroidectomia através da aplicação de trinitrato de glicerina tópica para alívio da dor autores Investigaram a eficácia na dor após hemorroidectomia e a relação entre dor e a pressão máxima de repouso, medidas no pré e no pós-operatório. Os autores não encontraram diferenças com o grupo controle, em relação à pressão máxima de repouso, mas a dor foi significantemente menor no grupo que utilizou trinitrato de glicerina tópica, assim como a pressão máxima de repouso foi significantemente menor. 20

Esperávamos que no período da dor maior após a hemorroidectomia a nifedipina pudesse diminuir, mesmo que de modo transitório, as pressões do canal anal; esse achado não foi encontrado, mas em relação à dor, houve diminuição dos valores, com conseqüente melhora da qualidade de vida no pós-operatório.

Apesar de encontrarmos, em nossa pesquisa, valores de pressão máxima de repouso dentro dos limites da normalidade (valores médios), chamou-nos a atenção que, em todos os grupos, existiam medidas isoladas, com aumento das pressões, restando a dúvida se existe ou não o espasmo esfincteriano na primeira semana de pós-operatório, mesmo que este espasmo seja ocasional. Esses achados de normalidade de pressão máxima de repouso também ocorreram em outra pesquisa realizada em nossa instituição, em que se estudou a diferença entre os valores manométricos, no pré e pós-operatório, de hemorroidectomia, mas neste caso as medidas foram obtidas com um mês de após a operação 7.

Apesar do uso de nifedipina se iniciar no primeiro dia de pós-operatório, já nas primeiras doze horas observamos diferença quanto aos valores de dor no período noturno. Isso seria explicado pela ação da nifedipina tópica já presente há horas.

Em relação aos outros momentos considerados observamos que até o quinto dia as médias de dor foram menores no grupo da nifedipina, mostrando ser a droga influente no combate à dor. No quinto e sexto dias de pós-operatório, além de dor espontânea, observamos diferenças em dor após evacuação, momento mais desconfortável do pós-operatório, como referiram os pacientes. Houve melhora da dor mas sua causa permanece indeterminada. Haveria melhora da perfusão tecidual que proporcionaria menor intensidade de dor? Haveria espasmo que diminuiria com a utilização dos bloqueadores de cálcio?

Em estudo sobre o efeito do trinitrato de glicerina tópica na cicatrização da ferida após a hemorroidectomia foram encontrados menores índices de dor 21. Nós observamos que no grupo que usou a nifedipina gel (Grupo 1) os índices de dor espontânea e após a evacuação foram menores. Isso justificaria o uso de nifedipina no pós-operatório, mesmo que não reduzisse significantemente as pressões do canal anal, como demonstrado nas medidas de manometria pré e pós-operatório. 
Em relação às pressões de repouso no primeiro dia após a operação, encontramos, aos três centímetros do orifício anal, três pacientes com pressões acima dos 70 centímetros de água; e aos dois centímetros, nove pacientes com pressões acima de $70 \mathrm{~cm}$ de água. Observando as médias das pressões máximas de repouso dos pacientes no pré e no pós-operatório, verificamos haver tendência às pressões serem maiores no primeiro dia do pós-operatório , mas não existem diferenças estatisticamente significante entre elas. Em relação às medidas de pressão máxima de repouso no quarto pós-operatório, também observamos diferenças estatisticamente não significantes, com um paciente, com pressão acima de $70 \mathrm{~cm}$ de água, no Grupo 1, e três, no Grupo 2. A diferença é não significante, mas, observamos uma tendência para maiores valores de pressão de repouso no Grupo dois.

No sétimo dia pós-operatório, observamos que a pressão máxima de repouso mostrou diferença próxima do nível de significância adotado, aos três cm do orifício anal, sendo bem maior o valor no Grupo dois (tabela 1). Esse resultado coloca em dúvida a existência de espasmo no pós-operatório e a possível ação da nifedipina gel na melhora desse espasmo.

A dor é um fenômeno subjetivo e sua avaliação na forma desse trabalho, embora seguisse regras aceitas de auto-avaliação, não acompanhou as alterações manométricas. Seria recomendável que houvesse uma casuística maior, mas a dificuldade em trabalhos clínicos dessa natureza impediu que conseguíssemos esse objetivo.

Portanto, medidas não cirúrgicas parecem óbvias no sentido de levar a melhor pós-operatório ,sem dano orgânico e risco de incontinência. Sabendo-se que , fisiologicamente, quedas de pressões anais ocorrem após a sexta década, não é impossível que pacientes submetidos a esfincterotomia na juventude possam cursar com incontinência fecal futura. Esta é a mais evidente justificativa da esfincterotomia química.

\section{CONCLUSÃO}

A avaliação manométrica anal de pacientes após hemorroidectomia, comparando as pressões de repouso, contração e esforço evacuatório antes e após o tratamento cirúrgico de hemorróidas pela técnica aberta com o uso da nifedipina gel $0,2 \%$, não demonstrou diferença estatisticamente significante. A intensidade de dor pós-operatória, porém, apresentou diferenças, demonstrando sua utilidade na analgesia .

Agradecimentos à Dra Ting Hui Ching pela orientação estatística desse trabalho.

\begin{abstract}
Introduction: Hemorrhoids are very common and pain following their surgical treatment causes great suffering. Various alternatives have been studied for reducing postoperative pain. Among these is surgical sphincterotomy, which may in some cases cause some degree of fecal incontinence. For this reason, several studies have used chemical sphincterotomy, with nifedipine, diltiazem, glycerin trinitrate or botulinum toxin. The objective of the present study was to investigate the effects of topical nifedipine for reducing anal canal pressures and consequently reducing postoperative pain. Material and method Topical gels of $\mathbf{0 . 2} \%$ nifedipine plus $2 \%$ lidocaine (Group 1) and $2 \%$ lidocaine alone (Group 2) were used following hemorrhoidectomy. Pressures were measured before the operation and on the first, fourth and seventh days after the operation. Pain was also evaluated on all of the first seven postoperative days using a visual analog scale. Results - There were no differences in relation to anal canal pressures, but lower pain levels were reported in the group that received nifedipine. Conclusion: Nifedipine gel was efficient for postoperative analgesia, but did not alter anal canal pressures.
\end{abstract}

Key words: Nifedipine, Manometry, Anal canal, Hemorrhoids/surgery, Postoperative pain, operative surgical procedures.

\section{REFERENCIAS}

1. Ho YH , Seow-Choen F, Low JY ,Tam M, Leong AP . Randomized controlled trial of trimebutine ( anal sphincter relaxant ) for pain after haemorrhoidectomy. Br J Surg 1997;84:377-9.

2. Khubchandani IT. Internal sphincterotomy with hemorrhoidectomy does not relieve pain. A prospective, randomized study. Dis Colon Rectum 2002;45(11)1452-7.
3. Amorotti C, Mosca D, Trenti C, Pintaud U. L'utilità della sfinterotomia interna laterale associata all'emorroidectomia sec. Milligan-Morgan: risultati di uno studio prospettico randomizzato. Chir Ital 2003;55(6):879-86.

4. Asfar SK, Juma TH, Ala-Edeen T. Hemorrhoidectomy and sphincterotomy : a prospective study comparing the effectiveness of anal stretch and sphincterotomy in reducing pain after hemorrhoidectomy. Dis Colon Rectum 1988;31:181-5. 
5. Galizia G, Lieto E, Imperatore V, Pelosio L, Castellano P. The usefulness of lateral internal sphincerotomy combined with hemorrhoidectomy in the treatment of hemorrhoids:a randomized prospective study. G Chir 2000;21(3):127134.

6. Altomare DF, Rinaldi M, Sallustio PL, Martino P, De Fazio M, Memeo V. Long-term effects of stapled haemorrhoidectomy on internal anal function and sensitivity. Br J Surg 2001;88:1487-91.

7. Cesar MAP, Ferretti CEA, Bassi DG, Machado Filho HN, Oliveira MF, Cesar RP, Speranzini MB. Efeito da Hemorroidectomia nas Pressoes do Canal Anal _ Estudo Manométrico. Rev Bras Coloproct, 2005;25(2):115-120

8. Chen HH. Anal manometric findings before and after hemorrhoidectomy: a preliminary report. Changgeng Yi Xue Za Zhi 1999;22:25-30.

9. Schouten WR, van Vroonhoven TJ. Lateral internal sphincterotomy in the treatment of hemorrhoids: a clinical and manometric study . Dis Colon Rectum 1986;29(6):86972.

10. Lolli P, Piccinelli D, Girardi S, Fasoli GL, Bettini P, Zago A, Rosa G. Quando associare la sfincterotomiainterna all'emorroidectomia. Ann Ital Chir 1995;66(6):809-12.

11. Hananel N, Gordon PH. Lateral internal sphincterotomy for fissure-in-ano - revisited. Dis Colon Rectum 1997; 40:597602.

12. Wiley M, Day P, Rieger N, Stephens J, Moore J. Open vs. closed lateral internal sphincterotomy for idiopathic fissurein-ano: a prospective, randomized, controlled trial. Dis Colon Rectum 2004;47(6):847-52.

13. Ortiz H, Marzo J, Armendariz P, De Miguel M. Quality of life assessment in patients with chronic anal fissure afetr lateral internal sphincterotomy. Br J Surg 2005;92(7):881-5.
14. Rosa G, Lolli P, Piccinelli D, Mazzola F, Zugni C, Ballarin A, Bonomo S. Calibrated lateral internal sphincterotomy for chronic anal fissure. Tech Coloproctol 2005;9(2):127-31; discussion 131-2

15. Mathai V, Ong BC, Ho YH . Randomized controlled trial of lateral internal sphincterotomy with haemorrhoidectomy. $\mathrm{Br}$ J Surg $1996 ; 83: 380-2$

16. Griffin N, Acheson AG, Jonas M, Scholefield JH. The role of topical diltiazem in the treatment of chronic anal fissures that have falied glyceryl trinitrate therapy. Int J Colorectal Dis 2002;4(6):430-5.

17. Perrotti P, Bove A, Antropoli C, Molino D, Antropoli M, Balzano A, De Stefano G, Attena F. Topical nifedipine with lidocaine ointment vs active control for treatment of chronic anal fissure. Dis Colon Rectum 2002;45(11):1468-75.

18. Ezri T, Susmallian S. Topical nifepidine vs. topical glyceryl trinitrate for treatment of chronic anal fissure. Dis Colon Rectum 2003;46(6):805-8.

19. Novell F, Novell-Costa F, Novell J. Topical glyceryl trinitrate in the treatment of anal fissure. Rev Esp Enferm Dig 2004;96(4):255-8.

20. Coskun A, Duzgun SA, Uzunkoy A, Bozer M, Aslan O, Canbeyli B. Nitroderm TTS (R) band application for pain after hemorrhoidectomy. Dis Colon Rectum 2001;4(5):680-5.

21. Hwang do Y, Yoon SG, Kim KY. Effect of 0,2 percent glyceryl trinitrate ointment on wound healing after a hemorrhoidectomy: results of a randomized, prospective, double-blind, placebocontrolled trial. Dis Colon Rectum 2003;46(7):950-4.

\section{Endereço para correspondência:} MARIA AUXILIADORA PROLUNGATTI CÉSAR

Avenida 9 de Julho, 441 / apto. 44

12020-200 - Taubaté - S.P. 